\title{
Mutant-Specific Gene Expression Profiling Identifies SRY-Related HMG Box 11b (SOX11b) as a Novel Regulator of Vascular Development in Zebrafish
}

\author{
Christopher E. Schmitt ${ }^{1,2,3}$, Melissa J. Woolls ${ }^{1,2,3}$, and Suk-Won Jin ${ }^{1, *}$
}

Previous studies have identified two zebrafish mutants, cloche and groom of cloche, which lack the majority of the endothelial lineage at early developmental stages. However, at later stages, these avascular mutant embryos generate rudimentary vessels, indicating that they retain the ability to generate endothelial cells despite this initial lack of endothelial progenitors. To further investigate molecular mechanisms that allow the emergence of the endothelial lineage in these avascular mutant embryos, we analyzed the gene expression profile using microarray analysis on isolated endothelial cells. We find that the expression of the genes characteristic of the mesodermal lineages are substantially elevated in the $\mathrm{kdrl}^{+}$cells isolated from avascular mutant embryos. Subsequent validation and analyses of the microarray data identifies Sox11b, a zebrafish ortholog of SRY-related HMG box 11 (SOX11), which have not previously implicated in vascular development. We further define the function sox11b during vascular development, and find that Sox11b function is essential for developmental angiogenesis in zebrafish embryos, specifically regulating sprouting angiogenesis. Taken together, our analyses illustrate a complex regulation of endothelial specification and differentiation during vertebrate development.

\section{INTRODUCTION}

Endothelial cells are a major component of the vascular system, which is essential for the development, growth, and survival of an individual. Failures in regulating the development of endothelial lineage contribute to a wide variety of pathological conditions, including cancer, psoriasis, arthritis, congenital or inherited diseases, as well as heart and brain ischemia, neurodegeneration, and osteoporosis (Carmeliet, 2005). During development, the endothelial lineage arises from mesodermal tis- sues. It has been reported that diverse mesodermal tissues including lateral plate mesoderm (Pardanaud et al., 1996), blood islands within the yolk sac (Ferkowicz and Yoder, 2005; Risau and Flamme, 1995), allantois (Caprioli et al., 2001), somitic mesoderm (Wilting et al., 1995), as well as placenta (Demir et al., 2007; Yamaguchi et al., 1993), can produce endothelial cells during development. Moreover, the entire mesoderm excluding notochord and prechordal mesoderm can serve as sources for endothelial cells (Noden, 1989), suggesting that angiogenic potential might be one of the intrinsic properties of the developing mesoderm. Subsequently, endothelial cells further differentiate as arterial, venous or lymphatic endothelial cells, each of which possesses unique molecular and cellular characteristics.

Specification and differentiation of the endothelial lineage are regulated by arrays of signaling pathways and transcription factors. Previous research has identified key signaling pathways that modulate the differentiation of the endothelial lineages from the nascent mesodermal progenitors, including vascular endothelial growth factor (VEGF) (Keck et al., 1989; Leung et al., 1989), fibroblast growth factor (FGF) (Abraham et al., 1986; Gospodarowicz et al., 1983), Wnt (Ishikawa et al., 2001), and Bone Morphogenetic Protein (BMP) (Yamashita et al., 1997), as well as essential transcription factors such as ETS transcription factor family member, Etv2/ER71 and FLI1 (Wernert et al., 1992), and T-cell acute lymphocytic leukemia protein 1 (TAL1) (Visvader et al., 1998). Given the developmental heterogeneity, it is not surprising that cellular responses to these factors vary among subsets of endothelial cells. For instance, we have recently reported that BMP2 signaling selectively activates venous endothelial cells without influencing arterial endothelial cells (Wiley and Jin, 2011). Similarly, Wnt signaling regulates the formation of the endothelial lineage within the tailbud mesoderm, without obvious effects on the lateral plate mesoderm (Martin and Kimelman, 2012). Therefore, identification of additional factors that regulate specifica-

\footnotetext{
${ }^{1}$ Yale Cardiovascular Research Center and Section of Cardiovascular Medicine, Department of Internal Medicine, Yale University School of Medicine, New Haven, CT, 06511, USA, ${ }^{2}$ Curriculum in Genetics and Molecular Biology, University of North Carolina at Chapel Hill, Chapel Hill, NC, 27599, USA, ${ }^{3}$ These authors contributed equally to this work.
}

*Correspondence: suk-won.jin@yale.edu 
tion and differentiation of the endothelial lineage will help us to further delineate the heterogeneity of endothelial cells.

To identify additional factors involved in vascular development, we have previously performed a large scale forward genetic screen using $T g(k d r l: e G F P)^{s 843}$ transgenic zebrafish, which labels all endothelial cells with eGFP (Jin et al., 2005). From the screen, we have identified a novel mutant, groom of cloche ( $g r c)$, which lacks the majority of the endothelial lineage at early stage (Jin et al., 2007), which is reminiscent of previously isolated mutant, cloche (clo) that lack both endothelial and hematopoietic lineages (Stainier et al., 1995). Despite the lack of endothelial cells at early stages, these avascular mutant embryos can generate endothelial cells at later stages (Jin et al., 2007), suggesting that distinct molecular mechanisms may be used to modulate the emergence of the endothelial lineage in these embryos.

In this report, we performed microarray analysis using the endothelial cells isolated from late stage avascular mutant embryos and compared the expression profile of transcription factors with endothelial cells isolated from wild-type embryos. We find that the expression level of 43 transcription factors is significantly up-regulated in endothelial cells isolated from avascular mutant embryos. The majority of transcription factors we identified in our microarray have not been implicated in vascular development. We further analyze the function of one of these transcription factors, SRY-related HMG Box 11b (Sox11b), in endothelial differentiation and subsequent vascular patterning. We find that Sox $11 \mathrm{~b}$ is expressed in endothelial cells during development, and is essential for sprouting angiogenesis in zebrafish. Our results demonstrate that developmental ontogeny of the endothelial lineage is far more complex than previously thought.

\section{MATERIALS AND METHODS}

Zebrafish husbandry and heatshock treatment

Zebrafish (Danio Rerio) embryos were raised as previously described (Westerfield, 1989). The following transgenic and mutant fish lines were utilized: $T g(k d r l: e G F P)^{s 843}$ (Jin et al., 2005), cloche (clo) $)^{55}$ (Stainier et al., 1995), Casanova (cas) ${ }^{\text {s4 }}$ (Alexander et al., 1998), groom of cloche (grc) ${ }^{\text {s635 }}$ (Jin et al., 2007), Tg(hsp70l:bmp2b) ${ }^{\text {tri3 }}$ (Chocron et al., 2007), Tg(hsp70l:

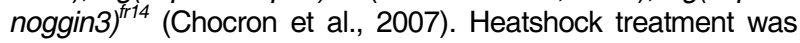
administered by incubating $24 \mathrm{hpf}$ embryos at $42^{\circ} \mathrm{C}$ for $30 \mathrm{~min}$.

Florescent activated cell sorting (FACS) and RNA isolation The 18.5hpf Tg(kdrl:eGFP) ${ }^{s 843}$ embryos were dissociated in HBSS with $5 \%$ FBS and subsequently incubated with 100 $\mu \mathrm{g} / \mathrm{ml}$ Liberase solution (Roche) for $15 \mathrm{~min}$ at $37^{\circ} \mathrm{C}$. Embryos were then triturated and the resulting suspension was pushed through a $40 \mu \mathrm{M}$ cell culture filter (BD Biosciences) and the reaction was stopped using $5 \mathrm{mM}$ EDTA, pH 8.0 in HBSS minus $\mathrm{Ca}^{2+}$ and $\mathrm{Mg}^{2+}$. Gates for flow cytometry were selected based on the Phycoerythrin versus FITC plot. Double sorts indicated an enrichment to $>95 \% \mathrm{GFP}^{+}$cells. RNA was extracted from isolated cells using Trizol (Invitrogen) and the accompanying protocol. Multiple rounds of flow cytometry were performed and RNA for each biological replicate was pooled. From the heatshock treated embryos, endothelial cells were harvested at $32 \mathrm{hpf}$.

Microarray analyses and quantitative RT-PCR

The WT ovation Pico Kit was used to amplify the RNA samples to satisfactory RNA integrity score (RIN) score (Schroeder et al.,
2006). Otherwise, gene expression profiling was performed as previously described (Lobenhofer et al., 2008) using an Agilent Zebrafish array version 2. Using the Statistical Analysis of Microarrays (SAM), the raw data for wild-type, grc, clo and cas was analyzed. We disregarded genes whose expression was down-regulated in cas, which would represent genes expressed in pharyngeal endoderm. Genes highly significantly up-regulated ( $q=0$, fold change $>2$ ) in both grc and clo mutants were further analyzed.

For qRT-PCR, RNA was extracted using the Qlagen RNeasy mini kit and accompanying protocol opting to add $300 \mathrm{ng}$ of carrier RNA to each sample. The iScript CDNA synthesis kit (Bio-Rad) was used to transcribe entire RNA extracts, immediately after RNA extraction. cDNA samples were then diluted to a volume of $300 \mu$ l. Using $2 X$ Power syber mastermix, $640 \mathrm{nM}$ of each primer, and $8 \mu \mathrm{l}$ of cDNA in a $25 \mu \mathrm{l}$ reaction, amplification of transcript amplicon was monitored on a Bio-Rad cfx96 system. Gene expression was normalized to either 18S rRNA or B-actin housekeeping genes. Melting curve analysis was performed on all reactions. $\mathrm{C}_{t}$ versus cDNA concentration plots were also used to determine that there was a linear ratio of amplification of housekeeping genes to gene-of-interest at a particular cDNA concentration. Data was analyzed using the $2^{-\Delta \Lambda C T}$ method (Livak and Schmittgen, 2001). At least three biological replicates of three technical replicates were performed for each conditon. Primers for qRT-PCR were generated using quantprime (REF\#890). Primers used were: 18s rRNA (5'-CACTTGTCCCTCTAAGAAGTTGCA-3' and 5'-GG TTGATTCCGATAACGAACGA-3'), sox11b (5'-CGAGTTCCC GGACTATTGCA-3' and 5' TCTCCCGCGATCATCTCACT-3'), zfhx4 (5'-CTCCTTTGTGTGGGAAGCAT-3' and 5'-CCCTG AATGTGGAACAGCAT- $3^{\prime}$ ), and klf5I (5'-AACCCGCAGTGAG AATCGCAAC-3' and 5'-ATCCATCTCCATCCGTGTCTGAGC$\left.3^{\prime}\right)$.

\section{In situ probe synthesis}

Probes were synthesized using the SP6/T7 DIG-UTP labeling kit (Roche) from linearized template. RNA was quantified, monitored by agarose gel electrophoresis for a singular product, diluted in in situ hybridization solution to $100 \mathrm{ng} / \mu \mathrm{l}$ and stored at $-20^{\circ} \mathrm{C}$.

\section{Morpholino knockdown of sox $11 b$}

Previously reported sox $11 \mathrm{~b}$ morpholino (5'-CATGTTCAAACA CACTTTTCCСTCT-3'), which blocks peptide synthesis, and control morpholino (5'-CCTCTTACCTCAGTTACAATTTATA$3^{\prime}$ ) were used (Veldman et al., 2007). All embryos were injected with $4.6 \mathrm{~nL}$ of injection mix containing $5 \mu \mathrm{M}$ HEPES, pH 7.6 and $0.05 \%$ Phenol red as a tracer.

\section{RESULTS AND DISCUSSION}

As previously reported, both clo and grc homozygous mutant embryos lack endothelial cells at $18 \mathrm{hpf}$ (Jin et al., 2007; Stainier et al., 1995) (data not shown). However, at $72 \mathrm{hpf}, \mathrm{kdrl}^{+}$ cells were present in these avascular mutant embryos (Figs. $1 \mathrm{~A}-1 \mathrm{C})$. Interestingly, counterstaining with DAPI in this experiment also showed that the midline region where are exclusively populated by $\mathrm{kdrl}^{+}$cells in wild-type embryos also contains a substantial number of $k d r l$ cells in avascular mutant embryos (Fig. 1A, yellow asterisks), alluding that vascular progenitors in these embryos may fail to undergo proper differentiation.

To better understand molecular mechanisms underlying the recovery of endothelial lineage, we analyzed the transcriptional 

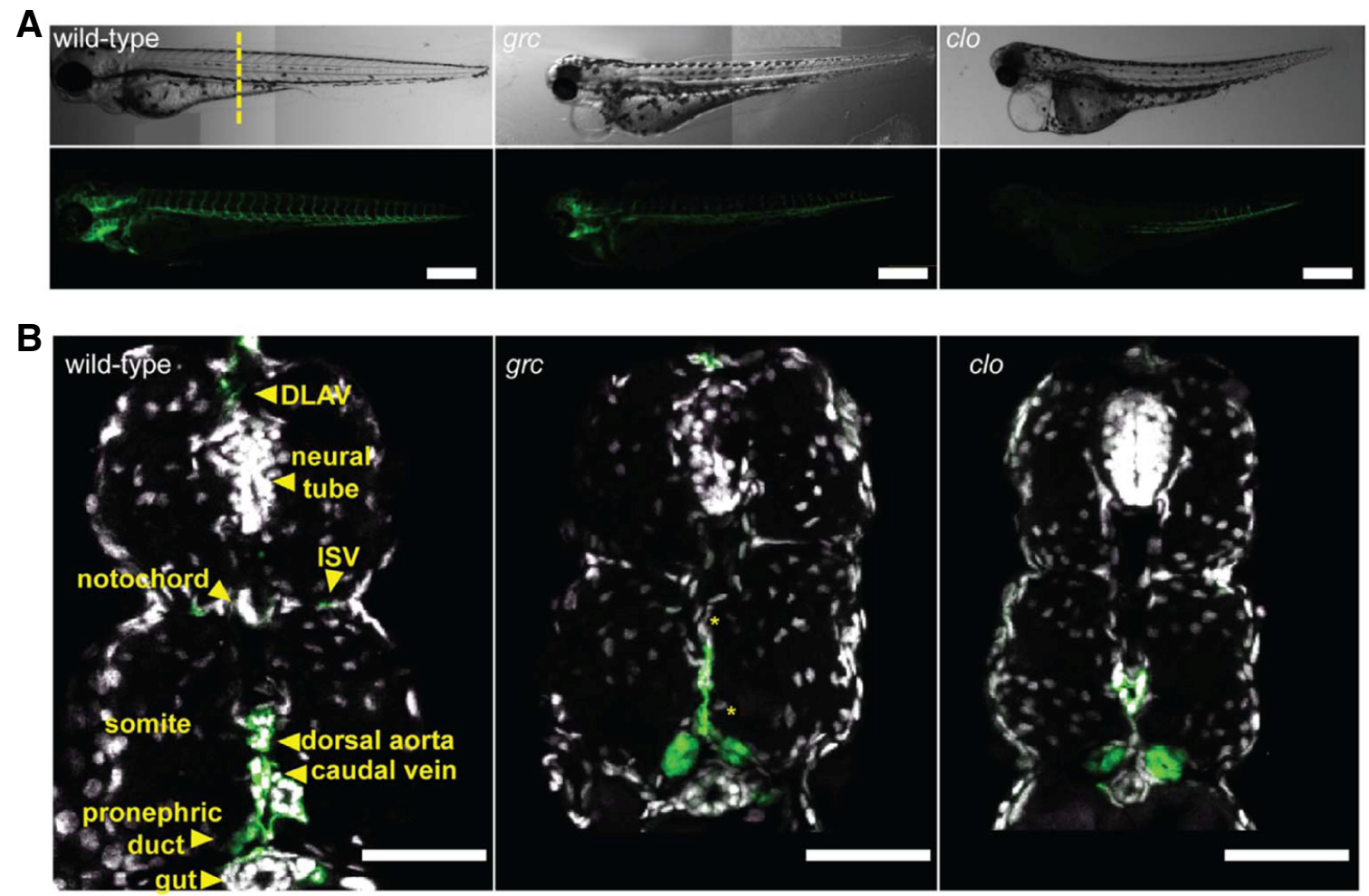

Fig. 1. Avascular mutant embryos generate endothelial cells at later stages. (A) Gross morphology of 72 hpf wild-type (left), groom of cloche ( $g r c)$ (middle), and cloche (clo) embryos in Tg(kdrl:eGFP) background. Both bright-field (top rows) and epifluorescent (bottom rows) images are shown. Scale bar $=250 \mu \mathrm{m}$. (B) Transverse section of $72 \mathrm{hpf}$ embryos taken from the area marked by dashed line in (A). GFP ${ }^{+}$endothelial cells are shown in green and nuclei stained with DAPI are shown in white. Scale bar $=50 \mu \mathrm{m}$.

profile of $\mathrm{kdrl}^{+}$cells in wild-type and avascular mutant embryos by microarray analyses (Fig. 2A). Since $k d r l$, a zebrafish ortholog of vascular endothelial growth factor receptor 2 (VEGFR2) (Bussmann et al., 2008), is also expressed in pharyngeal endoderm (Jin et al., 2005), it is possible that a significant portion of $k d r^{+}$cells isolated in avascular mutant embryos may represent non-endothelial lineage. Therefore, we used homozygous cas embryos wherein the entire presumptive endoderm fails to specify with little apparent effect on the vasculature (Jin et al., 2005). Genes down-regulated in $\mathrm{kdrl}^{+}$cells isolated from homozygous cas mutant embryos were discarded prior to further analyses and validation.

We found that the expression level of endothelial-enriched genes were largely unaltered in $\mathrm{kdrl}^{+}$cells of homozygous grc mutant embryos. In contrast, the majority of these genes were down-regulated in the same population from homozygous clo mutant embryos (Fig. 2A), suggesting that a locus affected by grc mutation may be only required in a subset of endothelial cells. A small subset of endothelial-enriched genes was markedly down-regulated in both homozygous grc and clo mutant embryos. For instance, we found that an arterial specific marker tbx20 (Ahn et al., 2000), as well as a putative zebrafish ortholog of mammalian Platelet Endothelial Cell Adhesion Molecule (PECAM), ENSDART00000084729, was significantly downregulated in $\mathrm{kdr}^{+}$cells isolated from both homozygous grc and clo mutant embryos (Fig. 2A). Interestingly, we found that genes up-regulated $(q=0)$ in all three mutants were found to be characteristic of other mesodermal, non-endothelial lineages such as somite, blood, or kidney (Fig. 2B). For instance, we found protein kinase $c$ delta a (prkcda), which are expressed in blood and somitic lineages (Patten et al., 2007), and adenosine kinase a (adka), which are expressed in blood and pronephric lineages (ZFIN direct data submission by Thisse et al., 2001), was up-regulated in $k d r^{+}$cells from avascular mutant embryos. Taken together, our microarray data suggest that $\mathrm{kdrl}^{+}$cells found in avascular mutant embryos may retain more mesodermal characteristics than those from wild-type embryos.

To better understand molecular characteristics of the $\mathrm{kdrl}^{\dagger}$ cells in avascular mutant embryos, we analyzed the expression level of transcription factors in our microarray data (Fig. 2C). We found that total of 43 transcription factors were up-regulated in $\mathrm{kdrl}^{+}$cells from avascular mutant embryos (Fig. 2D) at $\mathrm{q}=0$. Among these transcription factors, we further analyze the function of sox $11 b$, a zebrafish ortholog of SRY-related HMG Box 11 (SOX11) (Veldman et al., 2007), which is a member of SOXC subgroup (Bowles et al., 2000). Previously, it has been shown that Sox $11 \mathrm{~b}$ is essential for mediating retinal development and neuronal regeneration in zebrafish (Veldman et al., 2007). However, its role in endothelial cells and vascular development has not been investigated. Up-regulation of sox $11 \mathrm{~b}$ in $\mathrm{kdrl}^{+}$cells in avascular mutant embryos was confirmed by quantitative RT-PCR (Fig. 3A).

During development, sox $11 \mathrm{~b}$ is highly expressed in multiple tissues including neurons, somites, and retina as previously proposed. In addition, approximately at $24 \mathrm{hpf}$, sox $11 \mathrm{~b}$ expression was detectable in developing posterior axial vessels in 
A

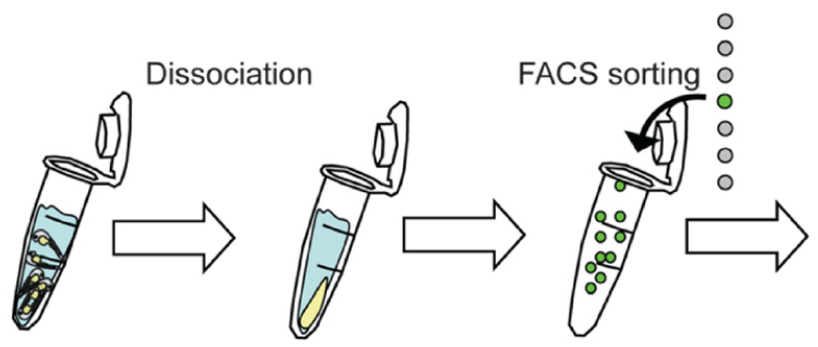

B

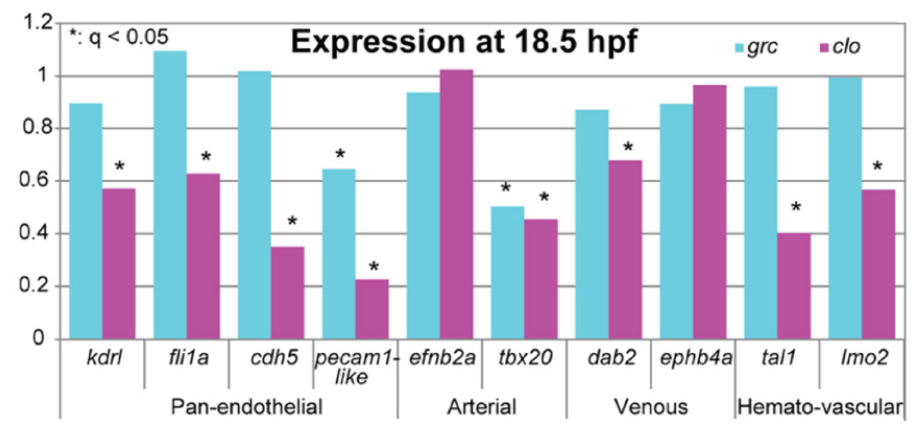

C

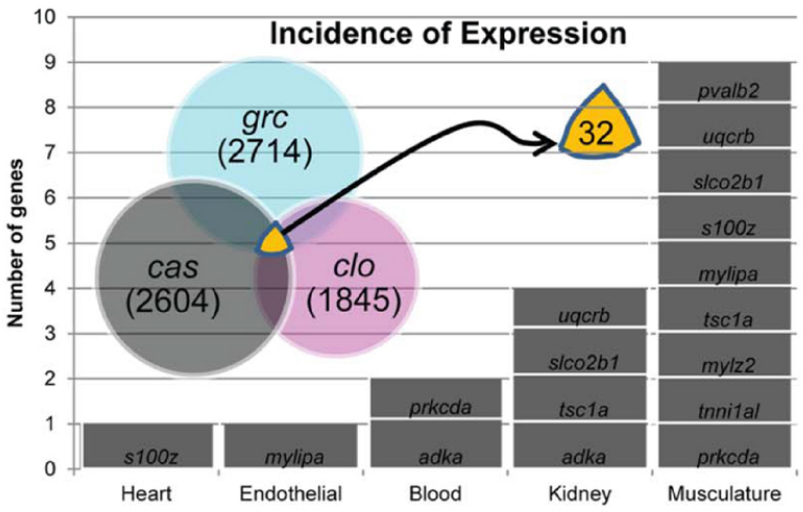

RNA extraction

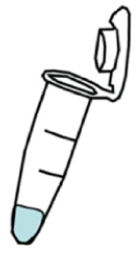

Microarray

D

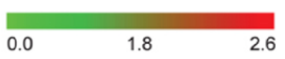

qRT-PCR

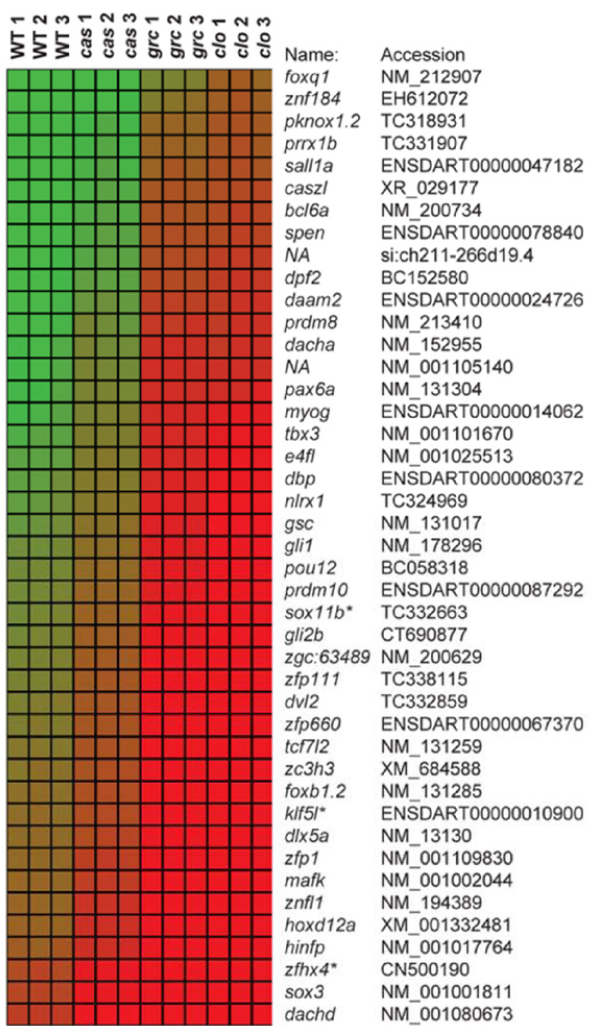

Fig. 2. Expression profile of $k d r^{+}$cells isolated from avascular mutant embryos. (A) Schematic diagram for molecular profiling. (B) Expression profile of known lineage specific markers in microarray ( $\left.{ }^{*} q<0.05\right)$. (C) Characteristics of genes which are up-regulated in endothelial cells in all three avascular mutant embryos. Total of 32 genes were shown to be up-regulated. (D) Expression profiles of putative transcription factors of which function have not previously implicated in the endothelial lineage. These genes were up in grc and $c l o(q=0)$, but not down-regulated in cas.

wild-type embryos (Fig. 3B). To analyze temporal changes in sox11b expression within endothelial cells, $\mathrm{kdrl}^{+}$cells were isolated from wild-type embryos and quantitative RT-PCR was performed. We found that sox $11 \mathrm{~b}$ expression can be detected as early as $18 \mathrm{hpf}$, and the level of expression gradually increases until $72 \mathrm{hpf}$ within endothelial cells, consistent with our in situ hybridization result (Fig. 3C). Interestingly, the expression of sox $11 \mathrm{~b}$ appears to be induced by bone morphogenetic protein (Bmp) signaling, as over-expression of Noggin3, an endogenous antagonist of Bmp signaling, led to a substantially decrease on the level of sox11b transcript level (Fig. 3D). Considering that Bmp signaling functions as a context-dependent pro-angiogenic cue (Kim et al., 2012; Wiley and Jin, 2011), it is possible that Sox11b may function as one of the effectors in this process.

To better assess the function of Sox $11 \mathrm{~b}$ during vascular development, we attenuated the activity of Sox $11 \mathrm{~b}$ by injecting morpholino (MO) anti-sense oligonucleotide as previously reported (Nasevicius and Ekker, 2000). Embryos injected with sox11b MO displayed discernible defects in vascular development, compared to control MO injected embryo (Fig. 4A). At 32 $\mathrm{hpf}$, the length of intersegmental vessels, which sprout from the dorsal aorta at this stage (Isogai et al., 2001), was substantially reduced in sox11b MO injected embryos (Fig. 4B). While 
A

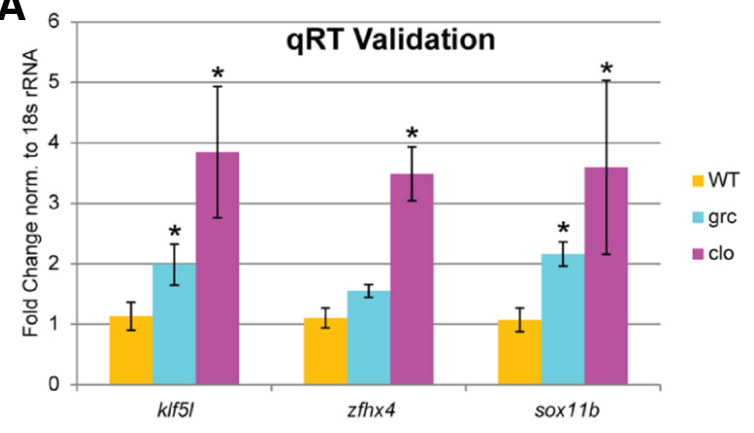

C

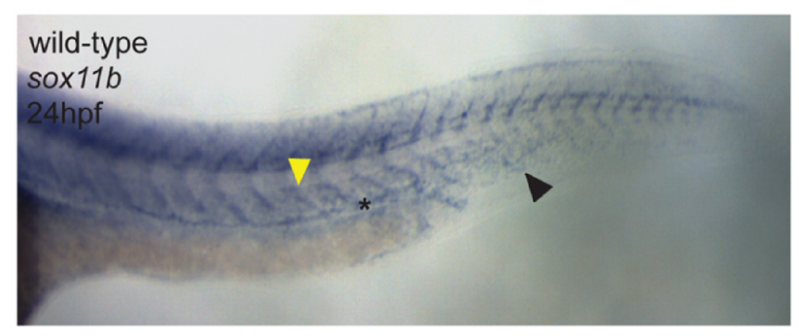

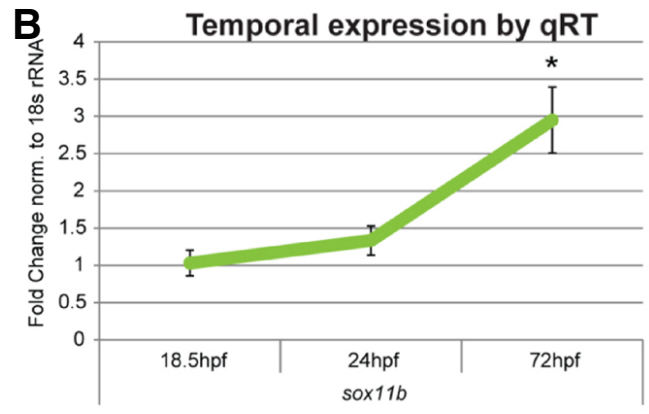

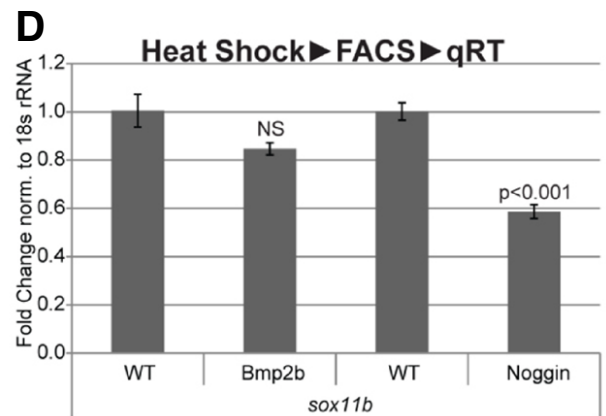

Fig. 3. Sox $11 \mathrm{~b}$ expression is elevated in $k d r^{+}$cells isolated from avascular mutant embryos. (A) Quantitative RT-PCR analyses confirmed the up-regulated expression of sox $11 \mathrm{~b}$ in endothelial cells of avascular mutant embryos. Two additional transcription factor, $k / f 5 /$ and $z f h \times 4$, were used as positive controls. (B) Temporal expression change of sox $11 \mathrm{~b}$ expression in endothelial cells. (C) In situ hybridization of sox $11 \mathrm{~b}$ at $24 \mathrm{hpf}$ wild-type embryo. In addition to neural tube and somite, axial vessels express sox11b. Anterior somite, axial vessel, and caudal vein are indicated by the yellow arrow, asterisk, and black arrow, respectively. (D) Effects of BMP signaling on sox $11 b$ expression. A decreased activity of BMP signaling significantly reduces the expression of sox $11 b$ in endothelial cells.

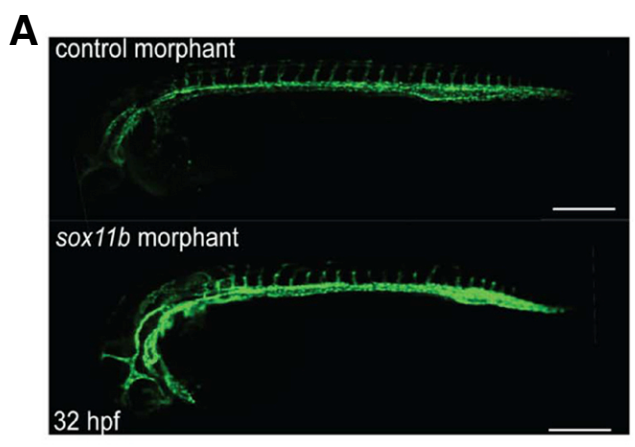

D

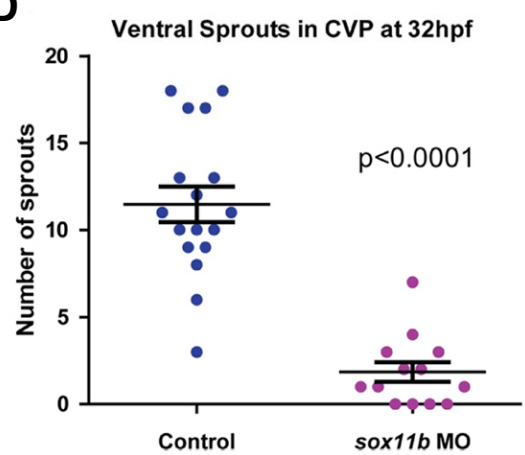

B

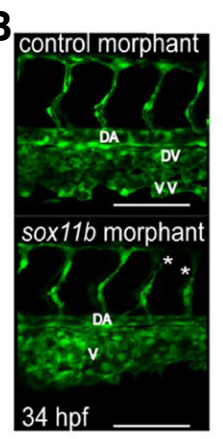

$\mathbf{E}$

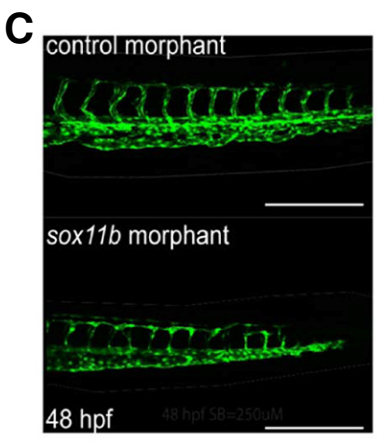

ISV length at $36 \mathrm{hpf}$

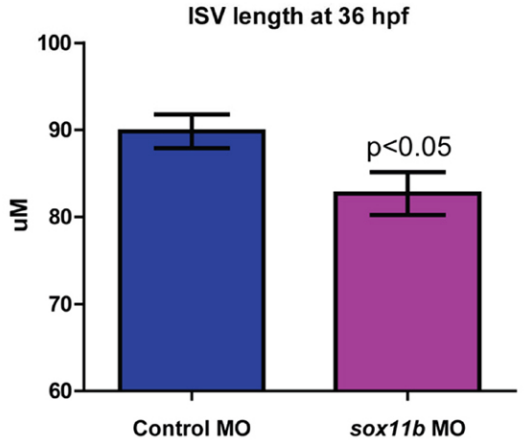

Fig. 4. Sox $11 \mathrm{~b}$ regulates sprouting angiogenesis during development. (A) Epifluorescent micrographs of control (top) or sox $11 \mathrm{~b}$ (bottom) morpholino (MO) injected embryos. Trunk regions posterior to the end of yolk extension are shown. Scale bar $=250 \mu \mathrm{m}$. (B) Intersegmental vessel and caudal vein defect in sox $11 \mathrm{~b} \mathrm{MO}$ injected embryos at $34 \mathrm{hpf}$. Scale bar $=100 \mu \mathrm{m}$. (C) Truncation of ISVs and plexus defects at $48 \mathrm{hpf}$. Scale bar $=250 \mu \mathrm{m}$. (D) Decreased venous sprouting angiogenesis in the caudal vein plexus (CVP) of sox $11 \mathrm{~b}$ MO injected embryos at $32 \mathrm{hpf}$ is quantified. (E) The effect of sox $11 \mathrm{~b} \mathrm{MO}$ on the length of intersegmental vessels is quantified ( $N=139, \mathrm{~N}=10$ embryos). 
control MO injected embryos had an average length of $89.82 \pm$ $1.92 \mu \mathrm{m}$ ( $\mathrm{N}=139$ ISVs), sox11b MO injected embryos intersegmental vessels were significantly shorter, $82.67 \pm 2.46$ $\mu \mathrm{m}(\mathrm{N}=156$ ISVs, $\mathrm{N}=8$ embryos; Figs. 4E and 4F), indicating that the function of Sox $11 \mathrm{~b}$ is essential for the morphogenesis of sprouting intersegmental vessels during development. Since a mammalian ortholog of Sox $11 \mathrm{~b}, \mathrm{SOX} 11$ is known to promote transcription of key cell cycle regulators including Cyclindependent kinase CDKN2B and Histones (Wang et al., 2010), as well as arrays of Actin binding proteins which modulate cell motility (Wang et al., 2010), it is possible down-regulation of Sox $11 \mathrm{~b}$ by MO injection led to a decreased endothelial proliferation and/or migration.

Since intersegmental vessels at $24 \mathrm{hpf}$ are arterial in nature (Isogai et al., 2001), we investigated whether Sox11b preferentially influences migration of arterial endothelial cells, by analyzing the effects of Sox11b knock-down on sprouting angiogenesis of caudal vein plexus (CVP). Previously, we reported that the CVP undergoes morphogenetic changes starting at $30 \mathrm{hpf}$ by forming extensive ventral sprouts (Wiley and Jin, 2011). In sox11b MO injected embryos, the number of angiogenic sprouts was drastically reduced compared to control $\mathrm{MO}$ injected embryos at $32 \mathrm{hpf}(1.85 \pm 0.56$ in sox $11 \mathrm{~b} \mathrm{MO}$ injected embryos and $11.4 \pm 1.0$ in control $\mathrm{MO}$ injected embryos; Figs. $4 \mathrm{G}$ and $4 \mathrm{H}$ ). Morphologically, the CVP in sox $11 \mathrm{~b} \mathrm{MO}$ injected embryos failed to undergo proper morphogenesis to generate the dorsal vein and the ventral vein as in wild-type embryos (Fig. 4G), reflecting the attenuated sprouting angiogenesis in these embryos.

Our results indicate that $\mathrm{kdrl}^{+}$cells in avascular mutant embryos express a unique transcriptional profile that allow them to circumvent the initial failure of endothelial specification, which led to the formation of rudimentary vascular structure in these embryos. We found that a number of transcription factors were selectively up-regulated in the $\mathrm{kdrl}^{+}$cells of avascular mutant embryos, indicating that these transcription factors may guide an alternative mechanism to generate the endothelial lineage. We analyzed the function of one of the transcription factors isolated from our microarray, Sox $11 \mathrm{~b}$, and found that Sox $11 \mathrm{~b}$ plays an important role in early morphogenesis of the vasculature. Taken together, our data provides a compelling evidence of developmental heterogeneity of the endothelial lineage.

\section{ACKNOWLEDGMENTS}

The authors would like to thank members of Jin lab for helpful discussion. This work has been supported by Developmental Biology Training Grant to C.E.S. and American Heart Association Pre-doctoral Fellowship to M.J.W., and grants from the NIH (HL090960) and the American Heart Association Scientist Development Award to S.-W.J.

\section{REFERENCES}

Abraham, J.A., Mergia, A., Whang, J.L., Tumolo, A., Friedman, J., Hjerrild, K.A., Gospodarowicz, D., and Fiddes, J.C. (1986). Nucleotide sequence of a bovine clone encoding the angiogenic protein, basic fibroblast growth factor. Science 233, 545-548.

Ahn, D.G., Ruvinsky, I., Oates, A.C., Silver, L.M., and Ho, R.K. (2000). tbx20, a new vertebrate T-box gene expressed in the cranial motor neurons and developing cardiovascular structures in zebrafish. Mech. Dev. 95, 253-258.

Alexander, J., Stainier, D.Y., and Yelon, D. (1998). Screening mosaic $\mathrm{F} 1$ females for mutations affecting zebrafish heart induction and patterning. Dev. Genet. 22, 288-299.

Bowles, J., Schepers, G., and Koopman, P. (2000). Phylogeny of the SOX family of developmental transcription factors based on sequence and structural indicators. Dev. Biol. 227, 239-255.

Bussmann, J., Lawson, N., Zon, L., and Schulte-Merker, S. (2008) Zebrafish VEGF receptors: a guideline to nomenclature. PLoS Genet. 4, e1000064.

Caprioli, A., Minko, K., Drevon, C., Eichmann, A., Dieterlen-Lievre, F., and Jaffredo, T. (2001). Hemangioblast commitment in the avian allantois: cellular and molecular aspects. Dev. Biol. 238, 64-78.

Carmeliet, P. (2005). Angiogenesis in life, disease and medicine. Nature 438, 932-936.

Chocron, S., Verhoeven, M.C., Rentzsch, F., Hammerschmidt, M. and Bakkers, J. (2007). Zebrafish Bmp4 regulates left-right asymmetry at two distinct developmental time points. Dev. Biol. 305, 577-588

Demir, R., Seval, Y., and Huppertz, B. (2007). Vasculogenesis and angiogenesis in the early human placenta. Acta Histochem. 109, 257-265.

Ferkowicz, M.J., and Yoder, M.C. (2005). Blood island formation: longstanding observations and modern interpretations. Exp. Hematol. 33, 1041-1047.

Gospodarowicz, D., Cheng, J., and Lirette, M. (1983). Bovine brain and pituitary fibroblast growth factors: comparison of their abilities to support the proliferation of human and bovine vascular endothelial cells. J. Cell Biol. 97, 1677-1685.

Ishikawa, T., Tamai, Y., Zorn, A.M., Yoshida, H., Seldin, M.F., Nishikawa, S., and Taketo, M.M. (2001). Mouse Wnt receptor gene Fzd5 is essential for yolk sac and placental angiogenesis. Development 128, 25-33.

Isogai, S., Horiguchi, M., and Weinstein, B.M. (2001). The vascular anatomy of the developing zebrafish: an atlas of embryonic and early larval development. Dev. Biol. 230, 278-301.

Jin, S.W., Beis, D., Mitchell, T., Chen, J.N., and Stainier, D.Y. (2005). Cellular and molecular analyses of vascular tube and lumen formation in zebrafish. Development 132, 5199-5209.

Jin, S.W., Herzog, W., Santoro, M.M., Mitchell, T.S., Frantsve, J., Jungblut, B., Beis, D., Scott, I.C., D'Amico, L.A., Ober, E.A., et al. (2007). A transgene-assisted genetic screen identifies essential regulators of vascular development in vertebrate embryos. Dev. Biol. 307, 29-42.

Keck, P.J., Hauser, S.D., Krivi, G., Sanzo, K., Warren, T., Feder, J., and Connolly, D.T. (1989). Vascular permeability factor, an endothelial cell mitogen related to PDGF. Science 246, 13091312.

Kim, J.D., Kang, H., Larrivee, B., Lee, M.Y., Mettlen, M., Schmid, S.L., Roman, B.L., Qyang, Y., Eichmann, A., and Jin, S.W. (2012) Context-dependent proangiogenic function of bone morphogenetic protein signaling is mediated by disabled homolog 2 . Dev. Cell 23, 441-448.

Leung, D.W., Cachianes, G., Kuang, W.J., Goeddel, D.V., and Ferrara, N. (1989). Vascular endothelial growth factor is a secreted angiogenic mitogen. Science 246, 1306-1309.

Livak, K.J., and Schmittgen, T.D. (2001). Analysis of relative gene expression data using real-time quantitative PCR and the 2(delta delta $C(T)$ ) method. Methods 25, 402-408.

Lobenhofer, E.K., Auman, J.T., Blackshear, P.E., Boorman, G.A., Bushel, P.R., Cunningham, M.L., Fostel, J.M., Gerrish, K., Heinloth, A.N., Irwin, R.D., et al. (2008). Gene expression res-ponse in target organ and whole blood varies as a function of target organ injury phenotype. Genome Biol. 9, R100.

Martin, B.L., and Kimelman, D. (2012). Canonical Wnt signaling dynamically controls multiple stem cell fate decisions during vertebrate body formation. Dev. Cell 22, 223-232.

Nasevicius, A., and Ekker, S.C. (2000). Effective targeted gene 'knockdown' in zebrafish. Nat. Genet. 26, 216-220.

Noden, D.M. (1989). Embryonic origins and assembly of blood vessels. Am. Rev. Respir. Dis. 140, 1097-1103.

Pardanaud, L., Luton, D., Prigent, M., Bourcheix, L.M., Catala, M., and Dieterlen-Lievre, F. (1996). Two distinct endothelial lineages in ontogeny, one of them related to hemopoiesis. Development 122, 1363-1371.

Patten, S.A., Sihra, R.K., Dhami, K.S., Coutts, C.A., and Ali, D.W (2007). Differential expression of PKC isoforms in developing zebrafish. Int. J. Dev. Neurosci. 25, 155-164.

Risau, W., and Flamme, I. (1995). Vasculogenesis. Annu. Rev. Cell Dev. Biol. 11, 73-91. 
Schroeder, A., Mueller, O., Stocker, S., Salowsky, R., Leiber, M., Gassmann, M., Lightfoot, S., Menzel, W., Granzow, M., and Ragg, T. (2006). The RIN: an RNA integrity number for assigning integrity values to RNA measurements. BMC Mol. Biol. 7, 3 .

Stainier, D.Y., Weinstein, B.M., Detrich, H.W., 3rd, Zon, L.I., and Fishman, M.C. (1995). Cloche, an early acting zebrafish gene, is required by both the endothelial and hematopoietic lineages. Development 121, 3141-3150.

Veldman, M.B., Bemben, M.A., Thompson, R.C., and Goldman, D. (2007). Gene expression analysis of zebrafish retinal ganglion cells during optic nerve regeneration identifies KLF6a and KLF7a as important regulators of axon regeneration. Dev. Biol. 312, 596-612.

Visvader, J.E., Fujiwara, Y., and Orkin, S.H. (1998). Unsuspected role for the T-cell leukemia protein SCL/tal-1 in vascular development. Genes Dev. 12, 473-479.

Wang, X., Bjorklund, S., Wasik, A.M., Grandien, A., Andersson, P., Kimby, E., Dahlman-Wright, K., Zhao, C., Christensson, B., and Sander, B. (2010). Gene expression profiling and chromatin immunoprecipitation identify DBN1, SETMAR and HIG2 as direct targets of SOX11 in mantle cell lymphoma. PLoS One 5, e14085.
Wernert, N., Raes, M.B., Lassalle, P., Dehouck, M.P., Gosselin, B. Vandenbunder, B., and Stehelin, D. (1992). c-ets1 proto-oncogene is a transcription factor expressed in endothelial cells during tumor vascularization and other forms of angiogenesis in humans. Am. J. Pathol. 140, 119-127.

Westerfield, M. (1989). The Zebrafish Book: A Guide for the Laboratory Use of Zebrafish (Brachydanio rerio), (Eugene, USA University of Oregon Press).

Wiley, D.M., and Jin, S.W. (2011). Bone morphogenetic protein functions as a context-dependent angiogenic cue in vertebrates. Semin. Cell Dev. Biol. 22, 1012-1018.

Wilting, J., Brand-Saberi, B., Huang, R., Zhi, Q., Kontges, G., Ordahl, C.P., and Christ, B. (1995). Angiogenic potential of the avian somite. Dev. Dyn. 202, 165-171.

Yamaguchi, T.P., Dumont, D.J., Conlon, R.A., Breitman, M.L., and Rossant, J. (1993). flk-1, an flt-related receptor tyrosine kinase is an early marker for endothelial cell precursors. Development 118, 489-498.

Yamashita, H., Shimizu, A., Kato, M., Nishitoh, H., Ichijo, H., Hanyu, A., Morita, I., Kimura, M., Makishima, F., and Miyazono, K. (1997). Growth/differentiation factor-5 induces angiogenesis in vivo. Exp. Cell Res. 235, 218-226. 